\title{
BMJ Open Impact of iron fortification on the geospatial patterns of malaria and non- malaria infection risk among young children: a secondary spatial analysis of clinical trial data from Ghana
}

\author{
Ashley M Aimone, ${ }^{1}$ Patrick Brown, ${ }^{1,2}$ Seth Owusu-Agyei, ${ }^{3}$ Stanley H Zlotkin, ${ }^{1,4}$ \\ Donald C Cole ${ }^{1}$
}

To cite: Aimone AM, Brown P, Owusu-Agyei S, et al. Impact of iron fortification on the geospatial patterns of malaria and non-malaria infection risk among young children: a secondary spatial analysis of clinical trial data from Ghana. BMJ Open 2017;7:e013192. doi:10.1136/ bmjopen-2016-013192

- Additional material is published online only. To view please visit the journal online (http://dx.doi.org/10.1136/10. 1136/bmjopen-2016-013192).

Received 25 June 2016 Revised 24 January 2017 Accepted 27 January 2017

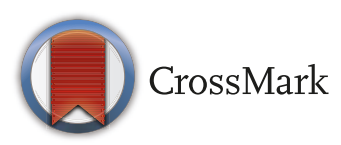

${ }^{1}$ Dalla Lana School of Public Health, University of Toronto, Toronto, Canada

${ }^{2}$ Departments of Analytics and Informatics, Cancer Care Ontario, Toronto, Canada ${ }^{3}$ Kintampo Health Research Centre, Kintampo, Ghana

${ }^{4}$ Centre for Global Child Health, Hospital for Sick Children, Toronto, Canada

Correspondence to Dr Ashley M Aimone; ashley.aimone@mail.utoronto.ca

\section{ABSTRACT}

Objectives Patterns of infection among children with varying levels of iron status in a malaria endemic area may vary spatially in ways requiring integrated infection and iron deficiency control programmes. The objective of this secondary analysis was to determine the geospatial factors associated with malaria and non-malaria infection status among young Ghanaian children at the end of a 5-month iron intervention trial.

Design Cluster-randomised controlled trial.

Setting Rural Ghana

Participants 1943 children (6-35 months of age) with geocoded compounds.

Interventions Point-of-use fortification with micronutrient powders containing vitamins and minerals with or without iron.

Primary and secondary outcome measures Generalised linear geostatistical models with a Matern spatial correlation function were used to analyse four infection response variables, defined using different combinations of inflammation (C-reactive protein, $\mathrm{CRP}>5 \mathrm{mg} / \mathrm{L}$ ) and malaria parasitaemia. Analyses were also stratified by treatment group to assess the independent effects of the iron intervention.

Results The by-group and combined-group analyses both showed that baseline infection status was the most consistent predictor of endline infection risk, particularly when infection was defined using parasitaemia. In the No-iron group, age above 24 months and weight-forlength z-score at baseline were associated with high CRP at endline. Higher asset score was associated with a $12 \%$ decreased odds of endline infection, defined as CRP $>5 \mathrm{mg} / \mathrm{L}$ and/or parasitaemia (OR $0.88,95 \%$ credible interval 0.78 to 0.98 ), regardless of group. Maps of the predicted risk and spatial random effects showed a defined low-risk area around the District centre, regardless of how infection was defined.

Conclusion In a clinical trial setting of iron fortification, where all children receive treated bed nets and access to malaria treatment, there may be geographical variation in the risk of infection with distinct high-risk and low-risk areas, particularly around municipal centres. Trial registration number clinicaltrials.gov, NCT01001871.
Strengths and limitations of this study

- The geostatistical analyses conducted in this study are the first of their kind to use model-based geostatistics with Bayesian inference and integrated nested Laplace approximations to explore the spatial variation and associated risk factors of malaria and non-malaria infection risk among children in rural Ghana after a 5-month randomised iron intervention trial.

- These analyses also provide input into the potential utility of geographical indicators, particularly for assessing infection risk potential, which could help guide the implementation of iron interventions in areas where infectious diseases are prevalent.

- Since satellite-derived spatial data often vary at a higher level than the individual (eg, village or region), the use of these data in statistical models with individual-level outcomes may increase the risk of a change of support or ecological inference problem.

- The use of C-reactive protein (CRP) as an indicator of non-malaria infection may have led to the underestimation of this outcome, since CRP is an acute phase protein that only rises in accordance with the first 48 hours of the inflammatory response.

- Straight-line distance, rather than distance by road, was used to estimate proximity to a health facility or the district centre, and thus may not have fully accounted for travel impedance.

\section{BACKGROUND}

The leading causes of death in children less than 5 years of age are infection related, and child mortality rates are highest in low-income and middle-income countries (LMICs). ${ }^{1}$ Malnutrition is also a large contributor to mortality ( $45 \%$ of all deaths), ${ }^{1}$ including micronutrient deficiencies. The most common nutritional disorder worldwide is iron deficiency, ${ }^{2}$ which is estimated to account for 2.2 million disability-adjusted life 
years lost per year among children less than 5 years of age. ${ }^{3}$ While iron deficient children are more vulnerable to infections (primarily due to compromised immune function), ${ }^{45}$ infection and inflammation can also affect iron homeostasis ${ }^{6}$ and the risk of iron deficiency. ${ }^{7}$ Iron status can usually be improved through food fortification or supplementation; however, evidence from a large randomised trial conducted in a malaria endemic area (Pemba, Zanzibar) indicated that supplementing young children with iron may increase their risk of malaria and infection-related morbidity and mortality, particularly if they are iron replete. ${ }^{8}$ Since iron supplements or fortificants would likely be withheld from children with malaria or other infections, assessing the risk of infection is an important component of developing safe and effective means of administering iron to children in LMICs.

Measuring biomarkers of infection status (such as C-reactive protein, CRP) among children in a low-resource context may have limited feasibility, due to the requirement of blood samples, and the potential for inflammation prevalence to vary over relatively short periods of time (eg, within seasons). As such, there is a need to identify indicators or risk factors associated with infection in LMICs that are more feasible to measure, and thus provide an efficient means of identifying high-risk populations. This need could be addressed with geographical factors (or 'geo-indicators'), such as the environmental or spatial characteristics of a village or region. ${ }^{9}$ Geo-indicators could provide additional insight into the dynamics and distribution of infection among children that informs treatment needs and the prophylactic use of iron supplementation or fortification. ${ }^{10-13}$ Collecting geospatial data is not invasive and comparatively less costly than biological measures, as geographical datasets are often publicly available on the internet. This also improves the access to and comparability of population-level statistics within and across national borders.

We previously conducted a secondary spatial analysis to determine the geospatial factors associated with infection status among iron deficient and sufficient children in rural Ghana. ${ }^{14}$ The results of these analyses suggested that the risk of infection may be related to elevation, and distance to the nearest health facility. The objective of the current analysis was to determine the geospatial factors associated with malaria and non-malaria infection status among Ghanaian children at the end of a 5-month randomised iron intervention trial.

\section{METHODS}

\section{Study population}

The data used in these analyses were generated from a study population of young children (6-35 months of age) who participated in a community-based cluster-randomised trial conducted in the Brong-Ahafo Region of Ghana in 2010. ${ }^{15}$ The trial consisted of a 5-month intervention period, where participants received micronutrient powders with or without iron, followed by a 1-month postintervention follow-up period (6 months in total). At the time of the trial, the estimated prevalence of malaria in Ghana was 7.2 million cases per year, and the prevalence of anaemia among preschool aged children was $76.1 \%$ (95\% CI $73.9 \%$ to $78.2 \%$ ). ${ }^{16}{ }^{17}$ Details of the clinical trial ${ }^{15}$ and geographical layout of the study area have been described elsewhere (Aimone, Brown, Zlotkin, Cole, Owusu-Agyei 2016).

\section{Measures from trial data}

Biological samples collected at the beginning and end of the 5-month intervention period were analysed for plasma ferritin (Spectro Ferritin S-22, Ramco Laboratories, Stafford, USA), CRP (QuickRead CRP, Orion Diagnostica, Espoo, Finland) and malaria parasite density (microscopy). ${ }^{15}$ Malaria screening was also performed on a weekly basis throughout the intervention period. If a child had a history of fever (within 48 hours) or an axillary temperature $>37.5^{\circ} \mathrm{C}$, a blood sample was drawn and analysed in the field via antigen test (Paracheck Pf), and in the lab using microscopy (thin and thick smears). Parasite density was combined with fever information to calculate clinical malaria incidence (episode counts). Demographic and nutrition-related information was also collected at baseline and included household assets, maternal education and child body weight and length. Weight-for-length and length-for-age z-scores were calculated using the WHO Child Growth Standards. ${ }^{18}$

\section{Variable preparation}

Geographical coordinates for the compounds of 1943 trial participants (representing 1539 clusters), surrounding health facilities and major road networks were collected using handheld global positioning system (GPS) units (WGS 1984 coordinate system, universal transverse Mercator zone 30N projection, EPSG code: 32630 ). Satellite-derived data were downloaded as global datasets ${ }^{19-21}$ and cropped according to the geographical boundaries of the trial area. Elevation had a range of 116-530 m, and values were centred by subtracting 250 . Land cover (LC) type consisted of three categorical values, representing woody savannah ( $\mathrm{LC}=8, \mathrm{n}=21 / 1943$ observations), urban and built up land (LC=13, n=243/1943 observations), and cropland/natural vegetation mosaic ( $\mathrm{LC}=14$, $\mathrm{n}=1679 / 1943$ observations). Normalized difference vegetation index (NDVI), a vegetation index included as a proxy for moisture, ${ }^{22}$ was averaged over the year that the trial was conducted (2010), and ranged in value from 0.22 to 0.62 . NDVI has also been used to create malaria risk distribution maps through the characterisation of vector habitat potential (eg, closed forest versus open forest). ${ }^{23}$ Two NDVI-LC interaction terms were created (NDVI*LC8 and NDVI*LC13) by overlaying the final NDVI and LC rasters, and masking the LC cells except where they had a value of 8 (or 13). These unmasked cells were given a value of zero.

Baseline age, in months, was calculated using the reported date of birth and trial enrolment date with a 
change point at 24 months. Household asset score was generated using a principal component analysis of six economic indicators (farm ownership, size and type of crops grown, type of toilet facility, and house ownership). Maternal education was included as a binary variable representing 'no' (0) versus 'any' (1) level of education. Baseline iron status was defined as serum ferritin concentration corrected for inflammation (baseline CRP) using a regression-based method (Namaste, Rohner, Huang, Bhushan, Flores-Ayala, Kupka, Mei, Rawat, Williams, Raiten, Northrop-Clewes, Suchdev, Adjusting ferrith concentration for inflammation, 2016, unpublished), and re-scaled by multiplying the corrected values by the inter-quartile range. Straight-line (Euclidean) distance to the nearest health facility was measured using the Near Table tool in ArcMap (ArcGIS V.10.2, Environmental Systems Resource Institute, Redlands, California, USA).

\section{Spatial modelling}

The endline data were analysed using generalized linear geostatistical models $(\mathrm{GLGM})^{24} 25$ with Bayesian inference via an Integrated Nested Laplace Approximation algorithm. ${ }^{26}$ Weak or uninformative priors were used for all model parameters with the exception of the Matern shape parameter (fixed at two). Four different endline infection outcomes were modelled separately: 1) inflammation (CRP $>5 \mathrm{mg} / \mathrm{L})$ with/without malaria parasitaemia; 2) inflammation (CRP $>5 \mathrm{mg} / \mathrm{L}$ ) without parasitaemia; 3) parasitaemia with measured concurrent fever (axillary temperature $>37.5^{\circ} \mathrm{C}$ ) or reported history of fever within 48 hours (ie, clinical malaria)) and 4) parasitaemia with or without concurrent fever or history of fever. All variables were binary-valued (coded as ' 1 ' for positive infection status at endline) and analysed using logistic regression, with the exception of the third definition (parasitaemia with fever), which was a count variable (number of new clinical malaria episodes during the intervention period) analysed using Poisson regression. Median infection probabilities (and 95\% credible intervals, CrIs) were modelled as the sum of the contributions of the independent variables, residual spatial variation, and a compound-level random effect term. The glgm function from the 'geostatsp' package in $\mathrm{R}$ was used for all spatial modelling. ${ }^{27} 28$

The spatial analyses were conducted in three modelling steps: 1) No-iron group only; 2) Iron group only; 3) both intervention groups combined. The interventions groups were analysed separately in order to differentiate the effects of time and the iron treatment. Infection probabilities from the combined-group models were plotted on a base map of the trial area, with study compounds, and major road networks. The maps depicted a spatial risk surface of predicted infection probabilities, which were computed as the posterior means of the odds or risk of infection. The posterior means were estimated assuming baseline values for individual-level covariates and location-specific values for spatial covariates. The posterior mean of the spatial random effect was also plotted, representing the residual spatial variation that corresponded to the difference between the predicted and expected odds or risk of infection at each location.

\section{Ethics}

The original clinical trial was approved by the Kintampo Health Research Centre (KHRC) Institutional Ethics Committee, the Ghana Health Service (GHS) Ethical Review Committee, the Hospital for Sick Children Research Ethics Board and the Food and Drugs Authority of Ghana. Approval for conducting the secondary analyses was obtained from the Hospital for Sick Children and University of Toronto Health Sciences Research Ethics Boards.

\section{RESULTS}

Baseline and endline characteristics of the study sample are presented in table 1 . A total of 1780 trial participants were included in the endline analyses, representing those with geocoded residences (compounds), who provided blood samples at endline and had both baseline and endline CRP and parasitaemia values (see online supplementary figure 1). For the infection outcome defined as parasitaemia with fever, there were 1939 observations included in the Poisson regression analysis, which corresponded to the number of children with geocoded compounds who had at least one recorded follow-up visit during the intervention period, and thus contributed data to the malaria count outcome.

The proportion of children with evidence of infection (elevated CRP and/or parasitaemia) increased from baseline to endline ( $37.0 \%$ at baseline vs $41.6 \%$ at endline). This increase may have been driven primarily by a greater prevalence of parasitaemia at endline $(27.1 \%$ vs $23.0 \%$ at baseline), though the difference between intervention groups was small $(26.3 \%$ vs $28.0 \%$ in the No-iron and Iron groups, respectively). The prevalence of non-malaria inflammation (CRP $>5 \mathrm{mg} / \mathrm{L}$ without parasitaemia) remained relatively stable over the course of the trial; however, the proportion in the Iron group was slightly higher at endline $(7.64 \%$ in the Iron group versus $6.58 \%$ in the No-iron group). After correcting ferritin concentration for inflammation (CRP) using the regression method, the prevalence of iron deficiency (ferritin $<12$ $\mu \mathrm{g} / \mathrm{L})$ was $25.5 \%(496 / 1943)$ at baseline and $12.7 \%$ $(226 / 1781)$ at endline $(95 / 886$ in the Iron group, $131 / 895$ in the No-iron group).

In the combined-group analyses, the risk of elevated CRP and/or parasitaemia at endline was negatively associated with household asset score and positively associated with baseline infection status (OR for definition 1: 0.88 , $95 \%$ CrI 0.78 to 0.98 ) (table 2). Baseline infection status was also significantly and positively associated with the corresponding endline outcome when defined as inflammation and/or parasitaemia (OR 1.84, 95\% CrI 1.36 to $2.50)$ or all parasitaemia $(2.75,95 \%$ CrI 1.91 to 3.95$)$. Although not statistically significant, the effect of the 
Table 1 Baseline and endline characteristics of the Ghana trial participants

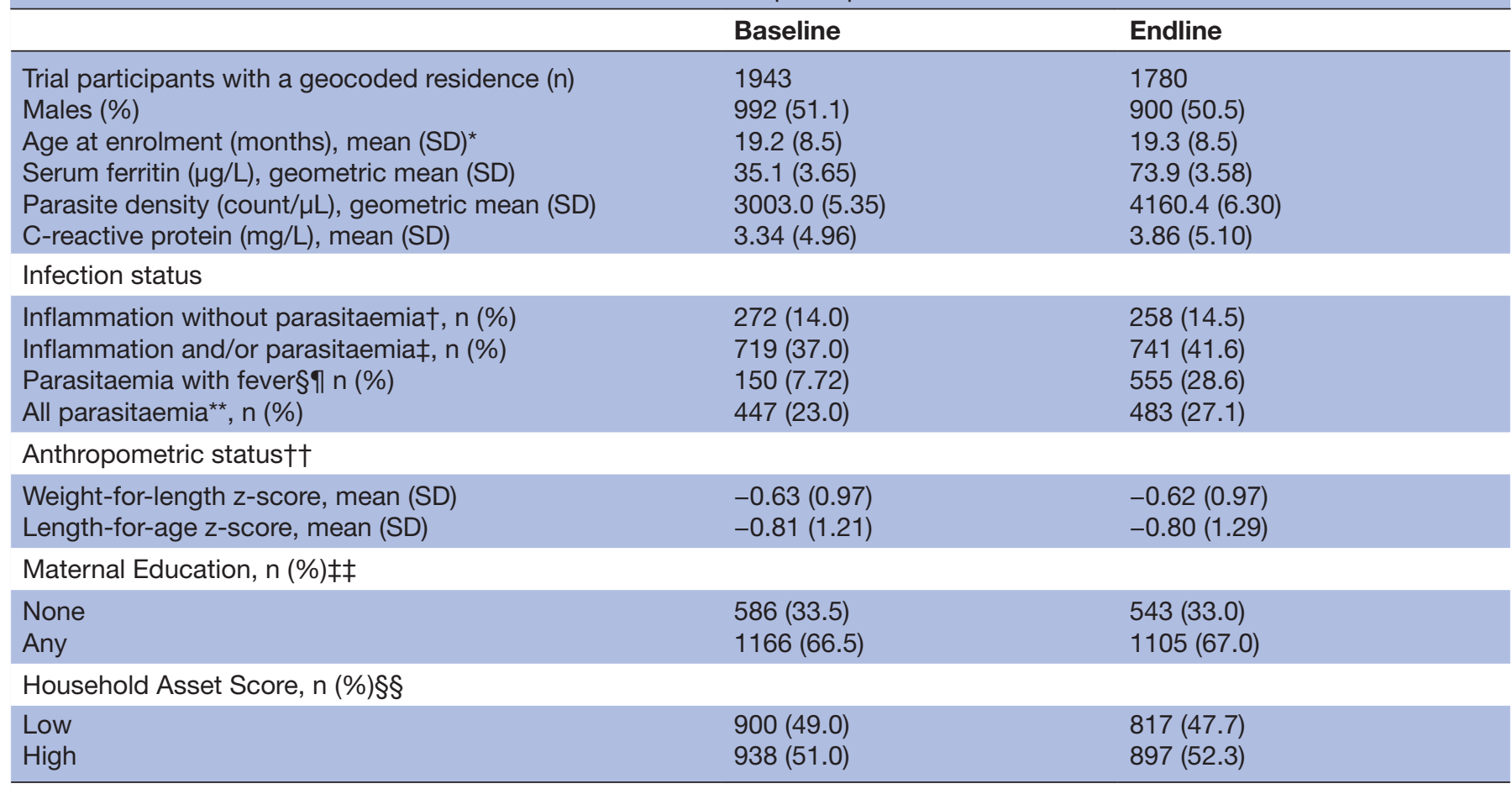

${ }^{*}$ Measured at baseline only.

${ }^{+}$Inflammation without parasitaemia $(n=1780)=C R P>5 \mathrm{mg} / \mathrm{L}$ without malaria parasitaemia.

${ }^{\ddagger}$ Inflammation and/or parasitaemia $(n=1780)=C R P>5 \mathrm{mg} / \mathrm{L}$ and/or any malaria parasitaemia.

${ }^{\S}$ Parasitaemia with fever $(n=1939)=$ any malaria parasitaemia with concurrent fever (axillary temperature $\left.>37.5^{\circ} \mathrm{C}\right)$ or history of reported fever (within 48hours).

१Endline value represents the period prevalence for 1939 participants.

${ }^{\star *}$ All parasitaemia $(n=1780)$ = any malaria parasitaemia (with/without fever).

††Measured at baseline; $z$-scores estimated using the WHO Child Growth Standards. ${ }^{23}$

¥†Measured at baseline only; total $n=1752$ (74 respondents were not mothers, 117 missing due to incomplete surveys).

$\S \S$ Measured at baseline only; Low= below median, High = above median; reduced sample size (approximately 1825) due to incomplete surveys and 'unknown' responses.

CRP, C-reactive protein.

'Group' variable seemed to suggest that the iron intervention was positively associated with endline infection risk, except in the case of outcome definition 3 (parasitaemia with fever) where the iron treatment may have had a protective effect.

Results from the by-group analyses indicated that the risk of elevated CRP (without parasitaemia) at endline among children in the No-iron group was positively associated with baseline weight-for-length z-score (OR $1.30,95 \%$ CrI 1.06 to 1.60 ) and negatively associated with age between 24 and 36 months (OR 0.92, 95\% CrI 0.84 to 1.006$)$. When infection was defined as all parasitaemia, baseline infection status became a significant factor (OR for definition 4: 2.86, 95\% CrI 1.97 to 4.17), indicating that children in the No-iron group with any parasitaemia (with or without CRP or fever) at baseline were up to $186 \%$ more likely to have parasitaemia at endline (see online supplementary table 1). In the Iron group, when infection was defined as inflammation and/ or parasitaemia, baseline infection status was the only factor associated with infection risk at endline (OR 2.27, $95 \%$ CrI 1.66 to 3.11). Similar results were found when infection was defined as all parasitaemia (OR 2.54, 95\% CrI 1.75 to 3.68) (see online supplementary table 2).

Plots of the predicted risk and residual spatial variation from all combined-group models are illustrated in figure 1 . The maps show a defined low-risk area around the District centre, regardless of how infection was defined. Conversely, the location of high-risk areas seemed to vary across models and, in some cases, roughly approximated elevation (figure 2). The maps depicting residual spatial variation also show defined high-risk and low-risk areas, indicating that a large amount of spatial variation was not explained by the variables included in the analyses and further supported the significant spatial random effects observed across all models.

\section{DISCUSSION}

The analyses presented herein explored the geospatial variation and associated factors of infection, defined using both inflammatory and parasitic biomarkers, among children in a malaria endemic area (rural Ghana) after a randomised iron intervention trial. Although none of the 


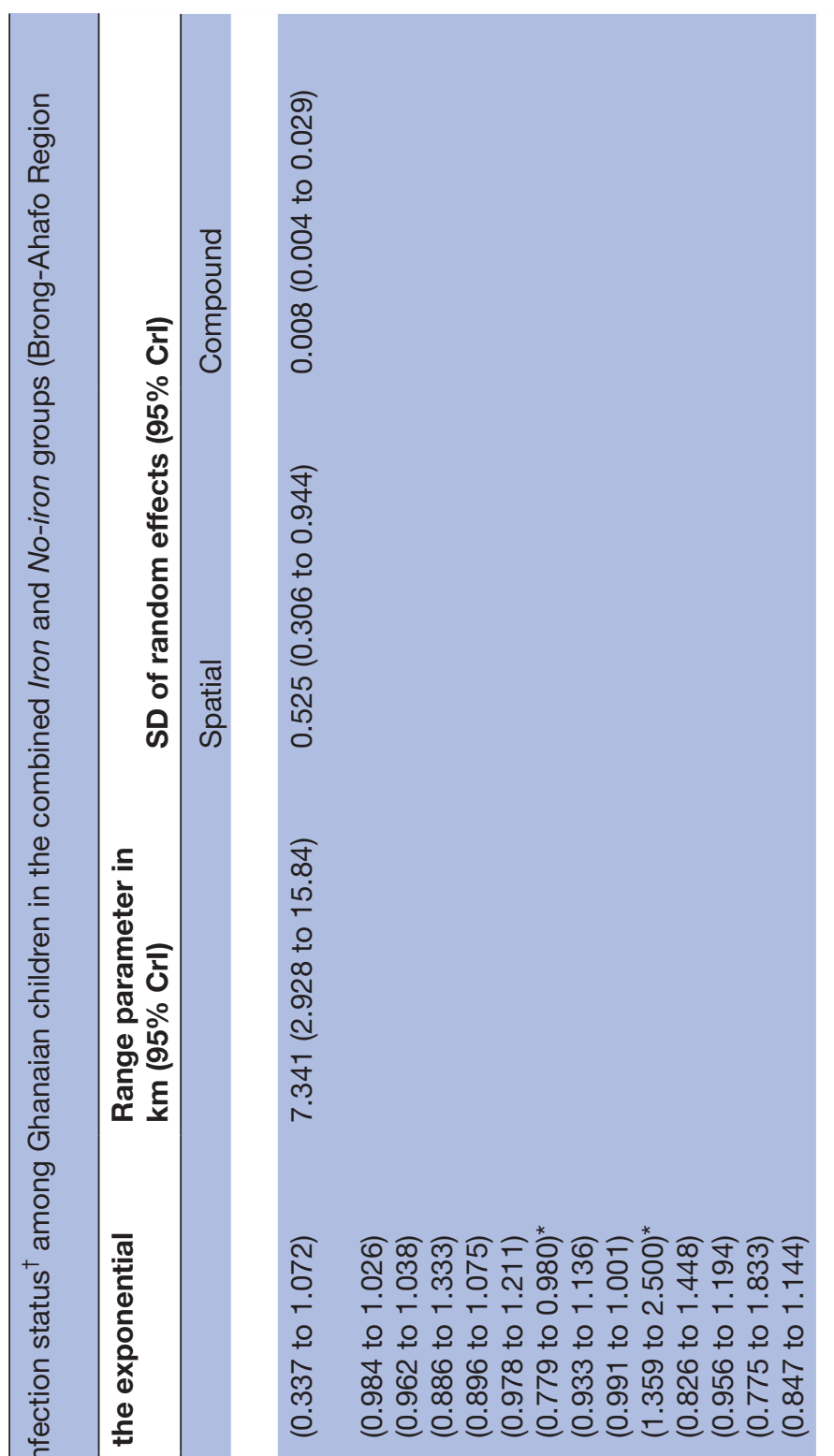

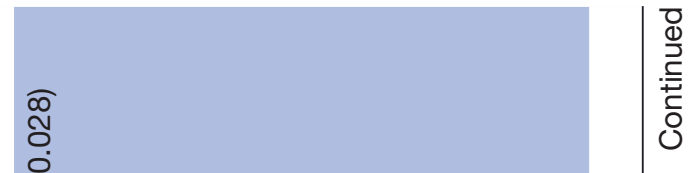

仓े

$\stackrel{\infty}{\infty}$

$\hat{\imath}$

운

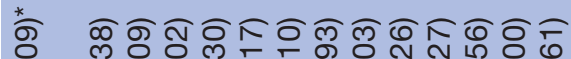
ले

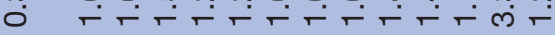
아웅ㅇㅇㅇㅇㅇㅇㅇㅇㅇㅇㅇㅇㅇㅇㅇㅇ

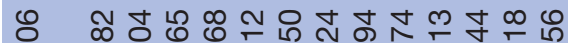

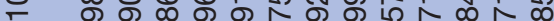

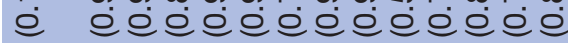




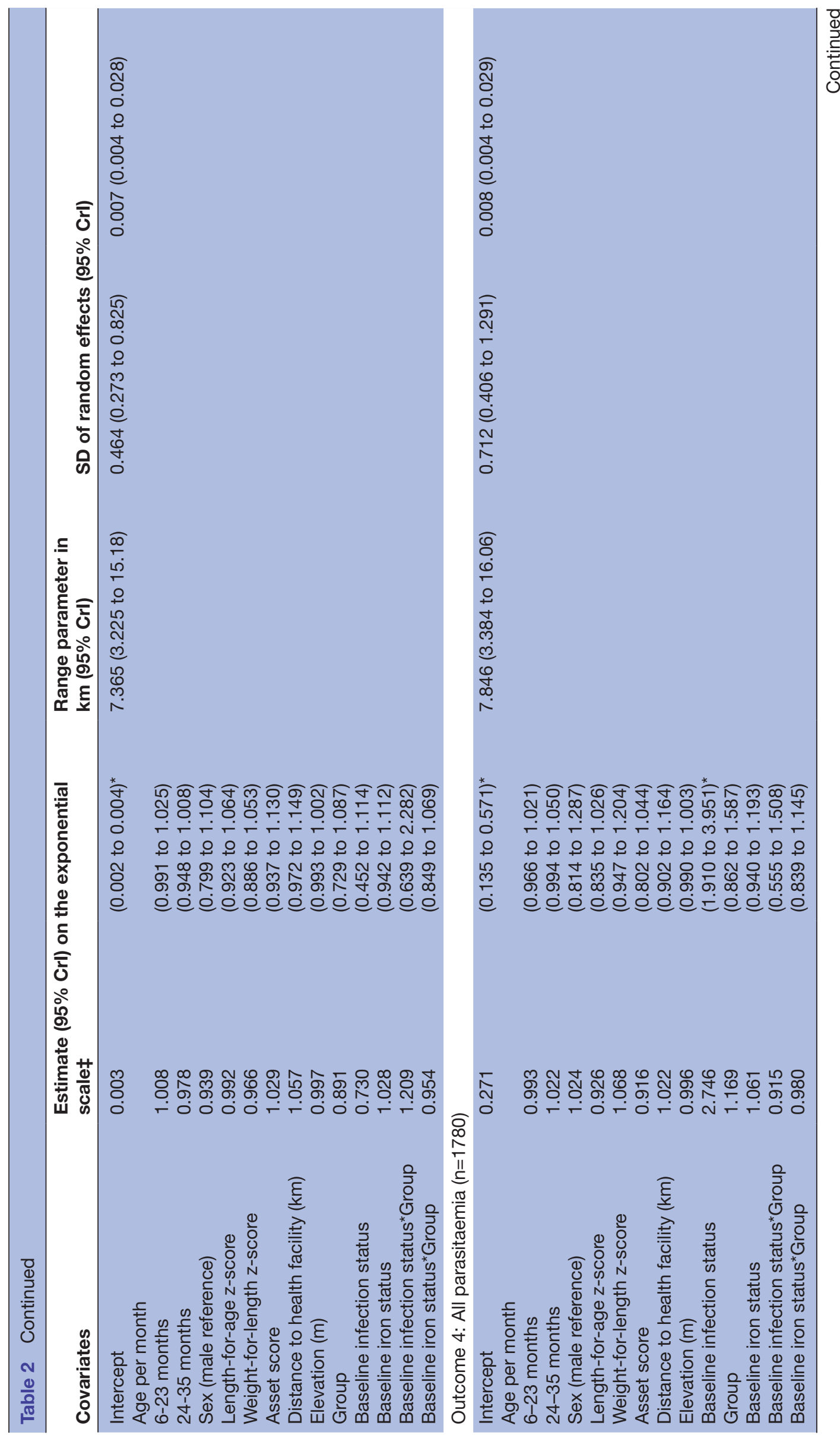


spatial variables included in the by-group or combinedgroup models demonstrated significant associations with endline infection status (regardless of how infection was defined), the plots of predicted infection probabilities and spatial random effects showed defined high-risk and low-risk areas across the study region, particularly around the District centre. In terms of individual-level effects, baseline infection status was a consistent predictor of endline infection risk, particularly in the Iron group and when infection was defined using parasitaemia (with or without inflammation).

The consistency of the relationship with baseline parasitaemia in both intervention groups is somewhat surprising, as current evidence might lead one to expect that providing iron to children with malaria would increase their risk of subsequent parasitic infections to a greater extent than those who do not receive iron. ${ }^{8}$ Interestingly, the Iron group analyses also revealed a significant effect of baseline infection status when acute non-malaria inflammation (high CRP without parasitaemia) was included in the definition. This observation is supported by recent research in mice demonstrating that iron supplementation may exacerbate non-malaria bacterial infections, and that coinfection with Plasmodium yoelli and Salmonella typhimurium can produce overwhelming Salmonella sepsis. ${ }^{29}$ Findings from the current analyses that also suggested a potential adverse effect of the iron intervention were the positive group effects across all infection definitions with the exception of clinical malaria (parasitaemia with fever). It should be noted, however, that these were non-significant associations only. This discrepancy is likely related to the fact that all children in both groups were monitored and treated for identified clinical malaria episodes throughout the intervention period. Therefore, any cases of asymptomatic malaria (parasitaemia without fever) may have persisted over the course of the trial, potentially interacting with the iron intervention as well as other opportunistic non-malaria infections.

After stratifying the analyses by group, we found that children in the No-iron group with a higher weight-for-length z-score (a measure of nutritional status) at baseline were $30 \%$ more likely to have high CRP $(>5 \mathrm{mg} / \mathrm{L})$ at endline (OR 1.30, 95\% CrI 1.06 to 1.60) (see online supplementary table 1). This positive association was not expected, given that well-nourished children (those with higher weight-length z-scores) are expected have a lower risk of infection. ${ }^{4}$ Since CRP becomes elevated during the first 48 hours of the inflammatory response, ${ }^{30}$ it is unlikely that anthropometric measures at baseline influenced inflammatory status 5 months later at endline. Rather, it is more likely that the association was influenced through another unmeasured factor, such as immune function. High CRP was also inversely associated with age in the No-iron group for children between 24 and 36 months (OR $0.92,95 \% \mathrm{CrI}(0.84$ to 1.00$)$ ), indicating an $8 \%$ lower odds of elevated CRP at endline for every month of age older. Assuming that elevated CRP without parasitaemia represented acute non-malaria infection, it is plausible 


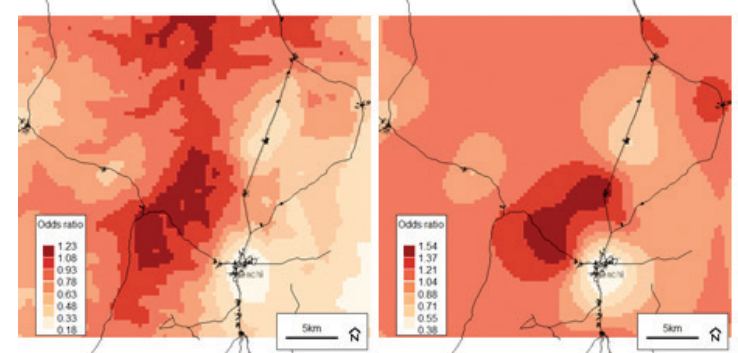

A

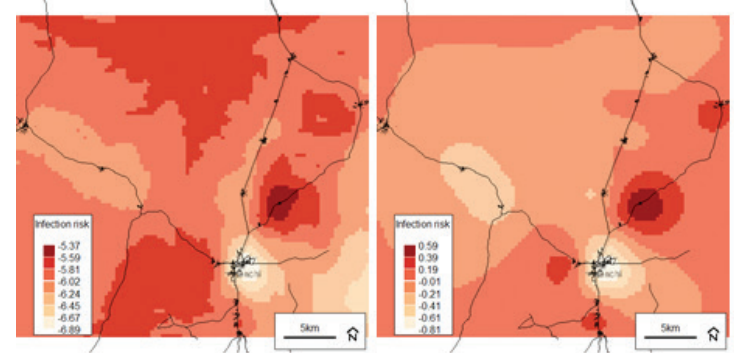

C

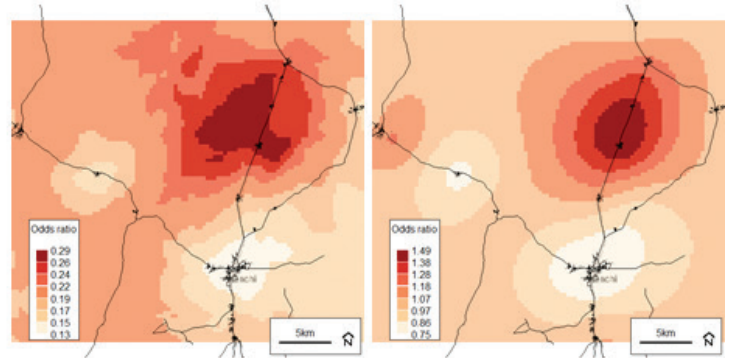

$\mathbf{B}$

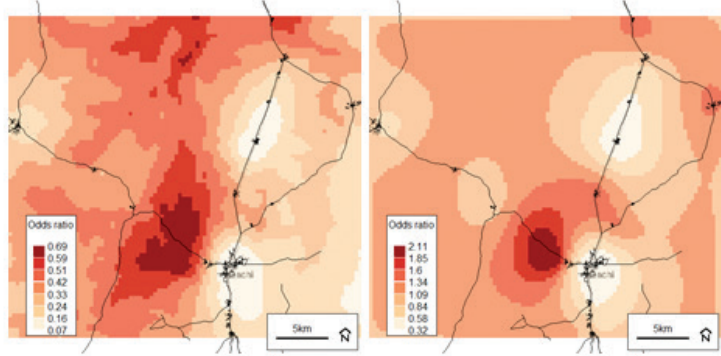

D

Figure 1 Predicted probabilities (left) and residual spatial variation (right) from the final combined-group models for the odds of inflammation (CRP $>5 \mathrm{mg} / \mathrm{L})$ and/or any malaria parasitaemia $(\mathbf{A})$; the odds of inflammation $(\mathrm{CRP}>5 \mathrm{mg} / \mathrm{L})$ without malaria parasitaemia (B); the risk of malaria parasitaemia with concurrent fever (axillary temperature $>37.5^{\circ} \mathrm{C}-$ or history of reported fever within 48 hours) $(\mathbf{C})$ and the odds of malaria parasitaemia with or without fever (D). Darker colour indicates higher risk at endline. Background (C) Stamen Design.

that the inverse relationship reflected the development of the immune system with age, thus resulting in older children being more resilient to infection exposure.

In the combined-group models (table 2), higher asset score was associated with a $12 \%$ decreased odds of endline infection, defined as CRP $>5 \mathrm{mg} / \mathrm{L}$ and/or parasitaemia (OR $0.88,95 \%$ CrI 0.78 to 0.98 ), suggesting that children from wealthier households had a lower risk of malaria or non-malaria infection at endline (regardless of whether they received micronutrient powders (MNPs) with or without iron). This finding was not surprising given the well-documented association between household wealth and child health in low-income and middle-income settings, ${ }^{31}$ however, the inconsistency of this relationship across infection definitions mav have been a reflection

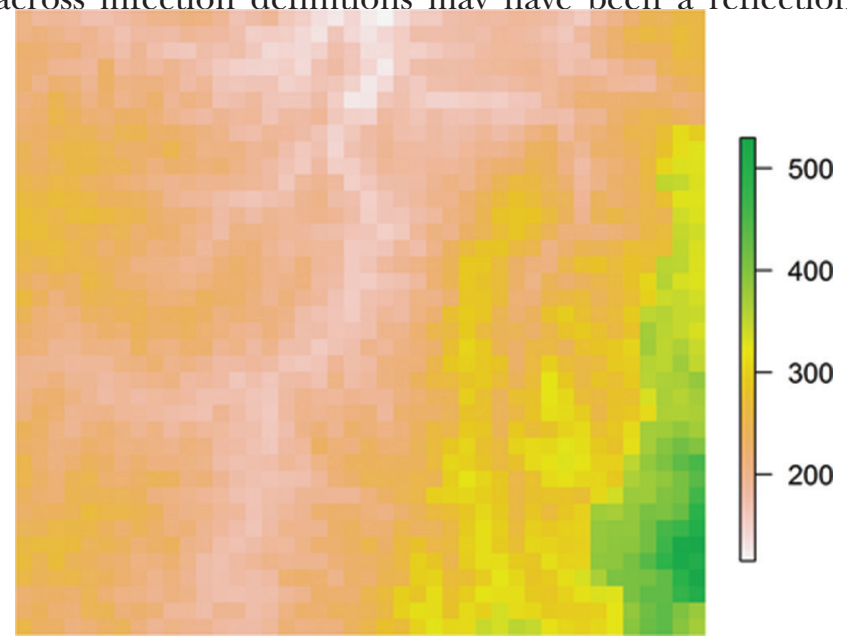

Figure 2 Elevation (metres) across the study area. Green colour indicates higher elevation. of other socioeconomic or behavioural factors that were not included in the analyses. ${ }^{32}$ Despite any differences in (or lack of) variable associations across infection outcomes, the residual spatial variation in infection risk remained significant in all combined-group models. The importance of accounting for residual spatial variation was further illustrated when the spatial random effects were plotted. For example, when infection was defined using CRP only, the model output plots looked similar (figure 1B), suggesting that the spatial random effect encompassed a large amount of the variation in predicted risk, while the variables included in the model did not.

For the infection outcomes that included parasitaemia, we observed similarities between the geographical variation in predicted mean risk and elevation (figure 2). This finding is consistent with our baseline analyses ${ }^{14}$ as well as other studies conducted in malaria endemic areas, where a higher prevalence of malaria is observed among populations living at lower elevations and vice versa. ${ }^{33-36}$ The association between malaria and elevation is related to temperature, as the early stages of parasite development are inhibited in colder environments, which are found at higher altitudes. ${ }^{37}$ Our baseline analyses also demonstrated a significant effect of distance to the nearest health facility, indicating that those children living farther from a health facility had an increased risk of malaria parasitaemia at baseline. The current endline analyses also suggested a potential risk-enhancing effect of distance to a health facility across all infection definitions, although the effect was not significant. This trend was further supported by the model output plots, which 
demonstrated larger low-risk areas around villages, and particularly around the District centre. Other studies have shown that living in a capital or municipal centre is associated with a reduced risk of adverse infection-related or nutrition-related health outcomes, ${ }^{38}{ }^{39}$ likely due to increased access to healthcare services and social amenities.

Overall, it appears that the effect of the intervention may have diminished the spatial associations observed at baseline; however, with the current data, it is difficult to differentiate between the effects of the iron intervention itself and the trial-related malaria mitigation activities (eg, universal provision of treated bed nets, and malaria treatment when indicated). Further research involving additional secondary analyses with expanded spatial datasets ${ }^{40}$ could shed more light on these relationships as well as their implications on the safety of iron interventions in malaria endemic areas. Until that time, the potential utility of geospatial analysis lies primarily in the assessment or planning stages of micronutrient programmes, in terms of where malaria and/or non-malaria infection risk is likely a concern, and thus where infection control efforts should be focused if iron fortification or supplementation is to be implemented.

A potential limitation of the present analyses was the use of straight-line (Euclidean) distance for estimating proximity to a health facility. While distance by road may have been a more appropriate indicator of travel impedance or access, incomplete or missing vector information (eg, miss-aligned junctions, missing or disconnected road segments) made it difficult to generate accurate measures. Nesbitt and colleagues (2014) compared different measures of travel impedance to estimate access to delivery care in the Brong-Ahafo region of Ghana ${ }^{41}$ and concluded that straight-line distance was as informative as distance by road for determining spatial access in rural Ghana. Therefore, we felt it was justified to use Euclidean distance in the present analyses.

Non-malaria infections were identified using CRP, an acute phase protein that rises in accordance with the early phase of the inflammatory response (approximately 48 hours). Other acute phase proteins (such as alpha-1acid glycoprotein) reach their peak concentration during the late phase (approximately $2-8$ days), which more closely approximates the change in ferritin concentration in response to inflammation. ${ }^{30}$ Since CRP was the only inflammatory biomarker available for the current analysis, and the original trial protocol was not designed to include diagnoses of other infection types, it is possible that the prevalence of non-malaria infection among participants was underestimated.

\section{CONCLUSION}

The analyses presented herein explored the spatial dynamics of infection risk in the context of an iron intervention trial in a malaria endemic area among children with varying levels of iron status, where all children received bed nets and access to malaria treatment. Geographical variation in the risk of malaria and non-malaria infections was observed with distinct highrisk and low-risk areas, particularly around the District centre. Overall, our findings emphasize the importance of considering geographical distribution when assessing infection risk and planning intervention trials in LMIC paediatric populations.

Acknowledgements The authors would like to acknowledge Seeba AmengaEtego and the members of the GIS team at the Kintampo Health Research Centre (Kintampo, Ghana) who collected the GPS data that were used in the secondary analysis.

Contributors AMA, SHZ and SO-A conducted the original trial in Ghana. AMA and SO-A coordinated the acquisition of geographical data. AMA conceived and conducted the secondary analysis with substantial contribution from PB. DCC and $\mathrm{SHZ}$ were also involved in the conception and design of the secondary analysis, and interpretation of data. AMA drafted the manuscript. All authors read and approved the final manuscript.

Funding Funding for this manuscript was provided by AMA's Doctoral Research Award from the Canadian Institutes of Health Research (ClHR).

Competing interests None declared.

Ethics approval Kintampo Health Research Center (KHRC) Institutional Ethics Committee, the Ghana Health Service (GHS) Ethical Review Committee, the Hospital for Sick Children Research Ethics Board and the Food and Drugs Authority of Ghana; University of Toronto Health Sciences Research Ethics Board.

Provenance and peer review Not commissioned; externally peer reviewed.

Data sharing statement Any unpublished data are available upon request by emailing the corresponding author.

Open Access This is an Open Access article distributed in accordance with the Creative Commons Attribution Non Commercial (CC BY-NC 4.0) license, which permits others to distribute, remix, adapt, build upon this work non-commercially, and license their derivative works on different terms, provided the original work is properly cited and the use is non-commercial. See: http://creativecommons.org/ licenses/by-nc/4.0/

(c) Article author(s) (or their employer(s) unless otherwise stated in the text of the article) 2017. All rights reserved. No commercial use is permitted unless otherwise expressly granted.

\section{REFERENCES}

1. WHO. Children: reducing mortality. fact sheet no 178. World Health Organization 2016.

2. WHO. Micronutrient deficiencies: iron deficiency anaemia. World Health Organization 2015.

3. Stoltzfus RJ. Iron deficiency: global prevalence and consequences. Food Nutr Bull 2003;24:S99-S103.

4. Katona P, Katona-Apte J. The interaction between nutrition and infection. Clin Infect Dis 2008;46:1582-8.

5. Raiten DJ, Sakr Ashour FA, Ross AC, et al; INSPIRE Consultative Group. Inflammation and nutritional science for programs/ Policies and interpretation of research evidence (INSPIRE). J Nutr 2015;145:1039S-108.

6. Spottiswoode N, Duffy PE, Drakesmith H, Iron DH. Iron, anemia and hepcidin in malaria. Front Pharmacol 2014;5:125.

7. Nemeth E, Tuttle MS, Powelson J, et al. Hepcidin regulates cellular iron efflux by binding to ferroportin and inducing its internalisation. Science 2004;306:2090-3.

8. Sazawal S, Black RE, Ramsan M, et al. Effects of routine prophylactic supplementation with iron and folic acid on admission to hospital and mortality in preschool children in a high malaria transmission setting: community-based, randomised, placebocontrolled trial. Lancet 2006;367:133-43.

9. Rezaeian M, Dunn G, St Leger S, et al. Geographical epidemiology, spatial analysis and geographical information systems: a multidisciplinary glossary. J Epidemiol Community Health 2007:61:98-102.

10. Schur N, Vounatsou P, Utzinger J. Determining treatment needs at different spatial scales using geostatistical model-based risk estimates of schistosomiasis. PLoS Negl Trop Dis 2012;6:e1773. 
11. Pullan RL, Gething PW, Smith JL, et al. Spatial modelling of soiltransmitted helminth infections in Kenya: a disease control planning tool. PLoS Negl Trop Dis 2011;5:e958.

12. Soares Magalhães RJ, Langa A, Pedro JM, et al. Role of malnutrition and parasite infections in the spatial variation in children's anaemia risk in northern Angola. Geospat Health 2013;7:341-54.

13. Sasaki S, Igarashi K, Fujino Y, et al. The impact of community-based outreach immunisation services on immunisation coverage with GIS network accessibility analysis in peri-urban areas, Zambia. $J$ Epidemiol Community Health 2011;65:1171-8.

14. Aimone AM, Brown PE, Zlotkin SH, et al. Geo-spatial factors associated with infection risk among young children in rural Ghana: a secondary spatial analysis. Malar J 2016;15:349.

15. Zlotkin S, Newton S, Aimone AM, et al. Effect of iron fortification on malaria incidence in infants and young children in Ghana: a randomized trial. JAMA 2013;310:938-47.

16. WHO. World malaria report 2009. Geneva, Switzerland: World Health Organization, 2009.

17. WHO. Worldwide prevalence of anaemia 1993-2005. WHO global database on anaemia. Geneva, Switzerland: World Health Organization, 2008.

18. WHO/UNICEF. WHO child growth standards and the identification of severe acute malnutrition in infants and children: a joint statement by the world health organization and the united nations children's Fund. Geneva, Switzerland: World Health Organization, 2009.

19. USGS . United States Geological Survey, 2012.

20. USGS. Land processes distributed active archive center, 2014.

21. Hengl T. Land cover types for 2010 based on the MODIS IGBP classe. SCenter LPDAA. ed. Worldgrids: (ISRIC - World Soil Information), 2013.

22. Samadoulougou S, Maheu-Giroux M, Kirakoya-Samadoulougou F, et al. Multilevel and geo-statistical modeling of malaria risk in children of Burkina Faso. Parasit Vectors 2014;7:350.

23. Guerra CA, Snow RW, Hay SI. A global assessment of closed forests, deforestation and malaria risk. Ann Trop Med Parasitol 2006;100:189-204

24. Diggle PJ, Tawn JA, Moyeed RA. Model-based geostatistics. Journal of the Royal Statistical Society: Series C 1998;47:299-350.

25. Diggle PJ, Ribeiro PJ. Model-based geostatistics. New York: Springer-Verlag, 2006.

26. Rue H, Martino S, Chopin N. Approximate bayesian inference for latent gaussian models by using integrated nested Laplace approximations. R Stat Soc Series B Stat Methodol 2009;71:319-92.

27. Brown PE. Model-Based geostatistics the easy way. J Stat Softw 2015;63:1-24.

28. R. R: a language and environment for statistical computing. Vienna, Austria: R Foundation for Statistical Computing, 2015.
29. Prestia K, Bandyopadhyay S, Slate A, et al. Transfusion of stored blood impairs host defenses against Gram-negative pathogens in mice. Transfusion 2014;54:2842-51.

30. Thurnham DI, McCabe GP. World Health Organization. Influence of infection and inflammation on biomarkers of nutritional status with an emphasis on vitamin A and iron. Report: Priorities in the assessment of vitamin $A$ and iron status in populations, Panama City, Panama, 15-17 September 2010. ed. Geneva: World Health Organization, 2012:63-80.

31. Black RE, Allen LH, Bhutta ZA, et al; Maternal and Child Undernutrition Study Group. Maternal and child undernutrition: global and regional exposures and health consequences. Lancet 2008;371:243-60.

32. Ezzati M, Utzinger J, Cairncross S, et al. Environmental risks in the developing world: exposure indicators for evaluating interventions, programmes, and policies. $J$ Epidemiol Community Health 2005;59:15-22.

33. Cohen JM, Ernst KC, Lindblade KA, et al. Topography-derived wetness indices are associated with household-level malaria risk in two communities in the western Kenyan highlands. Malar $J$ 2008:7:40.

34. Drakeley CJ, Carneiro I, Reyburn H, et al. Altitude-dependent and -independent variations in Plasmodium falciparum prevalence in northeastern Tanzania. J Infect Dis 2005;191:1589-98.

35. Brooker S, Clarke S, Njagi JK, et al. Spatial clustering of malaria and associated risk factors during an epidemic in a highland area of western Kenya. Trop Med Int Health 2004;9:757-66.

36. Reid H, Vallely A, Taleo G, et al. Baseline spatial distribution of malaria prior to an elimination programme in Vanuatu. Malar $\mathrm{J}$ 2010;9:150.

37. Hay SI, Omumbo JA, Craig MH, et al. Earth observation, geographic information systems and plasmodium falciparum malaria in subSaharan africa. Adv Parasitol 2000;47:173-215.

38. Shirayama Y, Phompida S, Shibuya K. Geographic information system (GIS) maps and malaria control monitoring: intervention coverage and health outcome in distal villages of Khammouane province, Laos. Malar J 2009;8:217.

39. Kandala NB, Madungu TP, Emina JB, et al. Malnutrition among children under the age of five in the democratic republic of Congo (DRC): does geographic location matter? BMC Public Health 2011;11:261.

40. Weiss DJ, Mappin B, Dalrymple U, et al. Re-examining environmental correlates of Plasmodium falciparum malaria endemicity: a dataintensive variable selection approach. Malar J 2015;14:68.

41. Nesbitt RC, Gabrysch S, Laub A, et al. Methods to measure potential spatial access to delivery care in low- and middle-income countries: a case study in rural Ghana. Int J Health Geogr 2014;13:25. 